\title{
Effective Mass of Bound and Resonant Two-Electron Pairs in a Simple Cubic Lattice
}

\author{
M. BAK* \\ Faculty of Physics, A. Mickiewicz University, Umultowska 85, 61-614 Poznań, Poland
}

\begin{abstract}
Effective masses $m^{*}$ of bound 2-electron pairs on a simple cubic lattice were investigated within tUWJ model within symmetry channels. Linear increase of $m^{*}$ with $|W|$ for intersite pairs and nonlinear behavior and sign change of $m^{*}$ in case of pairs with on-site component were found. $\left|m^{*}\right|$ turned out to be larger than twice free electron mass.
\end{abstract}

DOI: 10.12693 /APhysPolA.127.290

PACS: 03.65.Ge, 37.10.Jk

\section{Introduction}

A good candidate for mechanism of high temperature superconductivity (HTS) is the Bose condensation of tightly bound pairs. Thus the properties of single pair are a good starting point for investigations of HTS in the strong coupling limit. An important property is the pair's ability to move, described by its effective mass $m^{*}$. Although HTS materials are often considered two-dimensional (2D) they have in fact quasi-2D, strongly anisotropic 3D structure. There are also other strongly correlated 3D materials exhibiting superconductivity, like heavy fermion superconductors, Chevrel phases or doped $\mathrm{BaBiO}_{3}$ [1]. That warrants investigation of 3D systems. Current paper reports calculations of pair's effective mass within tUWJ model on "generic" simple cubic (sc) lattice.

\section{Formalism}

We shall start with tUWJ Hamiltonian $H=H_{0}+H_{\mathrm{I}}$ in standard denotations

$$
\begin{aligned}
H & =\sum_{\langle i, j\rangle} \sum_{\sigma} t_{i, j} c_{i, \sigma}^{\dagger} c_{j, \sigma}+U \sum_{\mathrm{I}} n_{i, \uparrow} n_{i, \downarrow} \\
& +\frac{W}{2} \sum_{\langle i, j\rangle} \sum_{\sigma, \sigma^{\prime}} n_{i, \sigma} n_{j, \sigma^{\prime}}+\frac{J}{2} \sum_{\langle i, j\rangle}\left(\boldsymbol{S}_{\mathrm{I}} \cdot \boldsymbol{S}_{j}-\frac{1}{4} n_{\mathrm{I}} n_{j}\right),
\end{aligned}
$$

where $\langle\ldots\rangle$ means summation over nearest neighbors (nn), $H_{0}$ - the hopping part - is the first term in the right hand side of the above equation, the rest describes interaction part $H_{\mathrm{I}}$. The solution of the Schrödinger equation $\left(H_{0}+H_{\mathrm{I}}\right)|\psi\rangle=E|\psi\rangle$ can be formally expressed as $|\psi\rangle=G_{0} H_{\mathrm{I}}|\psi\rangle$ with the Green function (GF):

$$
G_{0}=\left(E-H_{0}\right)^{-1}=\frac{1}{N} \sum_{\boldsymbol{k}} \frac{|\boldsymbol{k}\rangle\langle\boldsymbol{k}|}{E-E_{\boldsymbol{k}}},
$$

where $|\boldsymbol{k}\rangle$ symbolically denotes eigenstates of $H_{0}$, which are, in fact, the states of two particles with fixed center-of-mass momentum $\boldsymbol{K}$ and relative momentum $\boldsymbol{k}$ :

\footnotetext{
*e-mail: mbak@amu.edu.pl
}

$\left|\boldsymbol{K} \boldsymbol{k} \sigma_{1} \sigma_{2}\right\rangle=c_{\boldsymbol{K} / 2+k, \sigma_{1}}^{\dagger} c_{\boldsymbol{K} / 2-k, \sigma_{2}}^{\dagger}|0\rangle$. The eigenenergy of $H_{0}$ is given by

$$
\begin{aligned}
& E_{\boldsymbol{K}, \boldsymbol{k}}^{\mathrm{sc}}=-4 t\left(\cos \frac{K_{x}}{2} a_{x} \cos k_{x} a_{x}+\cos \frac{K_{y}}{2} a_{y} \cos k_{y} a_{y}\right. \\
& \left.\quad+\cos \frac{K_{z}}{2} a_{z} \cos k_{z} a_{z}\right),
\end{aligned}
$$

where $a_{x}, a_{y}, a_{z}$ are lattice constants. On the other hand, $H_{\mathrm{I}}$ is diagonal within the local orbitals basis in direct space. As we assume periodic boundary conditions the center of mass momentum $\boldsymbol{K}$ is conserved quantity, and we can introduce orthogonal "mixed" basis

$$
\begin{aligned}
& \left|\boldsymbol{K} \boldsymbol{r} \sigma_{1} \sigma_{2}\right\rangle= \\
& \quad \frac{1}{\sqrt{N}} \sum_{\boldsymbol{r}^{\prime}} \exp \left(\mathrm{i} \boldsymbol{K} \cdot\left(\boldsymbol{r}^{\prime}+\boldsymbol{r} / 2\right)\right) c_{\boldsymbol{r}^{\prime}, \sigma_{1}}^{\dagger} c_{\boldsymbol{r}^{\prime}+\boldsymbol{r}, \sigma_{2}}^{\dagger}|0\rangle,
\end{aligned}
$$

where $N$ is number of lattice sites and $\boldsymbol{r}$ denotes relative distance between electrons in a pair. We create singlets and triplets

$$
\begin{aligned}
& \left|S^{\boldsymbol{K}}(\boldsymbol{r})\right\rangle=(|\boldsymbol{K} \boldsymbol{r} \uparrow \downarrow\rangle \\
& -|\boldsymbol{K} \boldsymbol{r} \downarrow \uparrow\rangle) / \sqrt{2\left(1+\delta_{\boldsymbol{r}, 0}\right)}, \\
& \left|T_{0}^{\boldsymbol{K}}(\boldsymbol{r})\right\rangle=(|\boldsymbol{K} \boldsymbol{r} \uparrow \downarrow\rangle+|\boldsymbol{K} \boldsymbol{r} \downarrow \uparrow\rangle) / \sqrt{2}, \\
& \left|T_{1}^{\boldsymbol{K}}(\boldsymbol{r})\right\rangle=|\boldsymbol{K} \boldsymbol{r} \uparrow \uparrow\rangle, \\
& \left|T_{-1}^{\boldsymbol{K}}(\boldsymbol{r})\right\rangle=|\boldsymbol{K} \boldsymbol{r} \downarrow \downarrow\rangle .
\end{aligned}
$$

These states are eigenstates of $H_{\mathrm{I}}$ in tUWJ model; we will use it as a basis for expansion of $|\psi\rangle$. This way, we will obtain singlet $\psi_{\boldsymbol{K}}^{(s)}(\boldsymbol{r})=\left\langle S^{\boldsymbol{K}}(\boldsymbol{r}) \mid \psi\right\rangle$ and triplet $\psi_{\boldsymbol{K}, \zeta}^{(t)}(\boldsymbol{r})=$ $\left\langle T_{\zeta}^{K}(\boldsymbol{r}) \mid \psi\right\rangle$ wave function solutions of our 2-particle problem. For $\boldsymbol{r}$ 's larger than the range of Coulomb interactions $\left\langle S^{\boldsymbol{K}}(\boldsymbol{r})\left|H_{\mathrm{I}}\right| S^{\boldsymbol{K}}(\boldsymbol{r})\right\rangle=0$ (in the triplet case we use triplet bras and kets). Thus the nonzero "effective" part of $\left\langle H_{\mathrm{I}}\right\rangle$, hereafter denoted by $\boldsymbol{V}$, is small. For nn interactions it is given (in the singlet case) by $4 \times 4$ diagonal matrix with the elements $(U, W-J, W-J, W-J)$ on the diagonal. Both singlets and triplets mix states at " $r$ " 
with the states at " $-\boldsymbol{r}$ ", so in fact basis is spanned only by half of the sites of the direct space.

The singlet Green function is $4 \times 4$ matrix with elements

$$
\begin{aligned}
& G_{0}\left(E, \boldsymbol{K}, \boldsymbol{r}, \boldsymbol{r}^{\prime}\right)=\left\langle S^{\boldsymbol{K}}(\boldsymbol{r})\left|G_{0}\right| S^{\boldsymbol{K}^{\prime}}\left(\boldsymbol{r}^{\prime}\right)\right\rangle= \\
& 2 \delta_{\boldsymbol{K}, \boldsymbol{K}^{\prime}} \sum_{\boldsymbol{k}} \frac{\cos \boldsymbol{k r} \cdot \cos \boldsymbol{k} \boldsymbol{r}^{\prime}}{\left(E-E_{\boldsymbol{K}, \boldsymbol{k}}\right)\left(N \sqrt{1+\delta_{\boldsymbol{r}, 0}} \sqrt{1+\delta_{\boldsymbol{r}^{\prime}, 0}}\right)}, \\
& \quad \text { with } \boldsymbol{r}, \boldsymbol{r}^{\prime} \in\left\{\mathbf{0}, \boldsymbol{a}_{x}, \boldsymbol{a}_{y}, \boldsymbol{a}_{z}\right\} .
\end{aligned}
$$

The triplet GF has sin instead cos.

For $\boldsymbol{K}$ on some symmetry lines $G_{0}$ can be further simplified into the block-diagonal form by unitary transformation. The blocks transform according to irreducible representations of the point symmetry group of a lattice (irreps). For sc lattice ( $O_{h}$ group) the irreps are: $A_{1 \mathrm{~g}}$, traditionally called $s$-wave (if corresponding to the site 0 or $s^{*}$, i.e., "extended $s$ ", if corresponding to "layer" of nn sites $), E_{\mathrm{g}}\left(d_{x^{2}-y^{2}}\right.$ and $\left.d_{2 z^{2}-x^{2}-y^{2}}\right)$, and 3-dimensional $T_{1 \mathrm{u}}$, connected with three $p$-waves: $p_{x}, p_{y}$ and $p_{z}$. For $\boldsymbol{K}$ on $\mathrm{R} \Gamma \mathrm{MR}$ line $d_{x^{2}-y^{2}}$ decouples from other pairings (for anisotropic system also the other $d$-wave on $\mathrm{R} \Gamma \mathrm{M}$ lines). The GF for $p$-wave pairing is diagonal: all $p$ waves are decoupled from each other for arbitrary $\boldsymbol{K}$, yielding 3 degenerated scalar equations.

Eventually we are led to the matrix equation for the singlet case

$$
\tilde{\boldsymbol{\psi}}_{\boldsymbol{K}}=\tilde{\boldsymbol{G}}_{0}(E, \boldsymbol{K}) \tilde{\boldsymbol{V}} \tilde{\boldsymbol{\psi}}_{\boldsymbol{K}}
$$

Bold fonts denote that we are dealing with vectors and matrices, tilde - that the given quantity is after unitary transformation $\mathcal{U}$. Numerator of $\tilde{\boldsymbol{G}}_{0}=\mathcal{U} \boldsymbol{G}_{0} \mathcal{U}^{-1}$ consists of products of the functions: $1, \sqrt{2 / 3}\left(\cos k_{x}+\cos k_{y}+\cos k_{z}\right), \cos k_{x}-\cos k_{y}$, $\left(\cos k_{x}+\cos k_{y}-2 \cos k_{z}\right) / \sqrt{3}$, which transform according to $s, s^{*}, d_{x^{2}-y^{2}}$ and $d_{2 z^{2}-x^{2}-y^{2}}$ irreps respectively; $\tilde{\boldsymbol{V}}=\boldsymbol{V}$.

The condition for nontrivial solutions (of $E$ ) of Eq. (2) reads

$$
\operatorname{det}\left(\boldsymbol{I}-\tilde{\boldsymbol{G}}_{0}(E, \boldsymbol{K}) \tilde{\boldsymbol{V}}\right)=0,
$$

where $\boldsymbol{I}$ is identity matrix. Approximating $E$ around its minima by a free particle formula: $E-E_{\min }=$ $\lim _{\boldsymbol{K} \rightarrow 0} \hbar^{2} \boldsymbol{K}^{2} / 2 m^{*}$ we can get the effective mass $m^{*}$ of pairs in a given symmetry channel.

During calculations we take advantage of the formula

$$
\begin{gathered}
\frac{1}{\pi^{3}} \iiint_{0}^{\pi} \frac{\cos (p x) \cos (q y) \cos (n z) \mathrm{d} x \mathrm{~d} y \mathrm{~d} z}{E-a \cos x-b \cos y-c \cos z}= \\
\int_{0}^{\infty} \exp (-E t) I_{p}(a t) I_{q}(b t) I_{s}(c t) \mathrm{d} t,
\end{gathered}
$$

where $I_{n}(t)$ is modified Bessel function of the first kind [2].

\section{Results}

The dispersion curves for pairs of different symmetries for $W / t=-10.796$, i.e. for $d$-wave critical $W$ value [1], are shown in Fig. 1, together with parts of two-free electrons band (grayed out). Note the different number of curves in different parts of the Brillouin zone (BZ), a result of degeneration. On $\Gamma M$ line $d_{x^{2}-y^{2}}$-wave pairing is decoupled from $4 \times 4$ singlet determinant, so we have $1 d$-wave curve and 2 or 3 curves (for $U \geq 0$ and $U<0$, respectively) of mixed $s-d$ character. On $\Gamma R$ and $R M$ lines the $d$-wave curve is doubly degenerated on the expense of disappearing of one of $s$ - $d$ curves. The "shallow", close to the band bottom, solutions of singlet determinant in the $\Gamma$ point are " $d$-like", i.e., mostly $W-J$ dependent. Note their prominent degeneration at $R$ point in BZ.

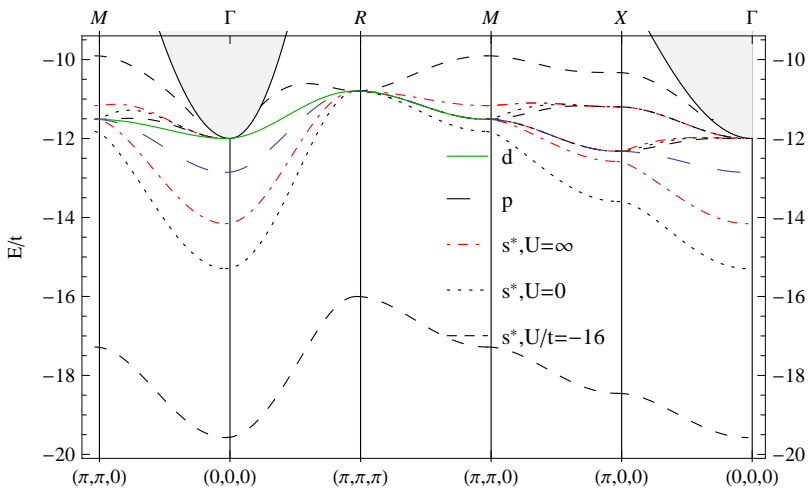

Fig. 1. Dispersion curves for $W-J=-10.796 t$.

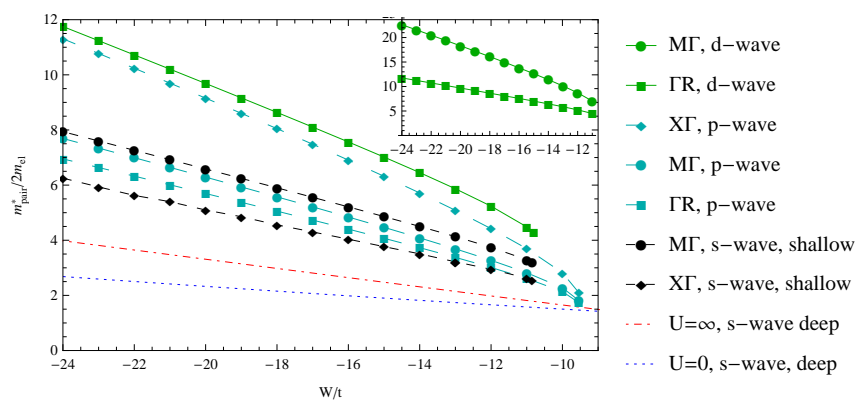

Fig. 2. $m_{\text {pair }}^{*} / 2 m_{e l}$ vs. $W / t, J=0$ for intersite pairs of various symmetries and symmetry directions in $\mathrm{Bz}$. Inset: $d$-wave for $M \Gamma$ and $\Gamma R$ lines.

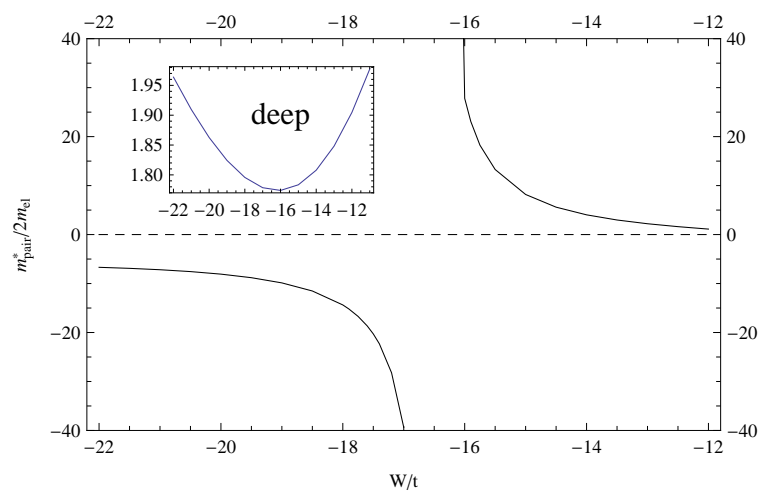

Fig. 3. $m_{\text {pair }}^{*} / 2 m_{e l}$ vs. $W / t, J=0, U=-16 t$ for on-site shallow pairs $\left(s^{*}\right)$. Deep pair in the inset.

The respective effective masses (in the units of two freeelectron masses) are shown in Fig. 2. The masses increase 
linearly with $|W-J|$. The anisotropy of the states in various symmetry directions is well shown. Only the strongly bound $s^{*}$ states for $U 0$ are isotropic.

For $U<0$ effective masses are isotropic and depend on $W$ non-linearly as shown in Fig. 3. The effective mass of "shallow" states, closer to band bottom, with smaller binding energy is diverging around $W \sim U$, indicating the change of curvature of $E(K)$ relation. The "deep" states, with $E \sim U$ reach minimal $m^{*}$ around $W=U$.

\section{Conclusions}

Dispersion curves and effective masses $m^{*}$ of bound 2-electron pairs on sc lattice (in the limit of empty lattice) in the tUWJ model were found in symmetry channels. $W$ and $J$ always appear together as $W-J$ - they are equivalent. $m^{*}$ increase with $|W-J|$ for intersite pairs, while non-linear behavior with $W-J$ of (strongly bound, extended $s^{*}$-wave) pairs with significant on-site component is found. Effective masses of pairs are larger than masses of two free electrons. $m^{*} / 2 m_{e l}$ can be close to one for shallow $s^{*}$-wave states and is the largest $(\approx 25)$ in the case of $d$-wave pairs on $\Gamma R$ direction. The calculations in $2 \mathrm{D}$ models suggest that considering second-neighbor hopping might decrease $m^{*}[3]$; similar effect could be reached by considering anisotropic lattice [4].

\section{References}

[1] R. Micnas, J. Ranninger, S. Robaszkiewicz, Rev. Mod. Phys. 62, 113 (1990).

[2] G.F. Koster, J.C. Slater, Phys. Rev. 96, 1208 (1954).

[3] M. Bak, Acta Phys. Pol. A 97, 209 (2000).

[4] P.E. Kornilovitch, J.P. Hague, arXiv:1407.3040. 